\title{
Adhärenz transparent gemacht
}

In der immunmodulatorischen Basistherapie der Multiplen Sklerose (MS) kann eine unzureichende Adhärenz den Therapieerfolg gefährden. Autoinjektoren wie der elektronische Injektor RebiSmart ${ }^{\mathrm{Tm}}$ können die Adhärenz verbessern, machten Dr. Antonios Bayas, leitender Oberarzt und Leiter der MS-Ambulanz, Klinikum Augsburg, deutlich.

Häufige Gründe für eine mangelnde Adhärenz in der MS-Therapie sind neben grippeartigen Nebenwirkungen und lokalen Injektionsreaktionen eine Spritzenmüdigkeit sowie das Vergessen von Injektionen. „Um einen Therapieerfolg zu sichern, ist es daher wichtig, eine unzureichende Adhärenz zu erkennen und in diesem Falle Gegenmaßnahmen einzuleiten“, sagte Bayas. Aus Marktanalysen wisse man, dass sich nach einem Zeitraum von drei Monaten nur etwa $70 \%$ und nach einem Jahr nur noch 40-60\% der Patienten ihr MS-Therapeutikum spritzen. Mit dem elektronischen Injektor RebiSmart ${ }^{\mathrm{Tm}}$ könnten anhand der Speicherfunktion objektive Adhärenzdaten in größerem Umfang leicht dokumentiert werden, erklärte Bayas.
Der MS-Fachmann referierte die Ergebnisse einer zwölfwöchigen Studie mit dem elektronischen Injektor, in der $88 \%$ der 119 Patienten mindestens $80 \%$ und $67 \%$ alle der geplanten Injektionen applizierten [Lugaresi et al. BMC Neurology 2012; 12: 7]. Die Adhärenz korrelierte dabei nicht mit dem Behinderungsgrad gemäß EDSS, der Kognition gemäß PASAT oder der Vortherapie, ergänzte Bayas. Eine Verbesserung der Adhärenz durch den RebiSmart ${ }^{\text {TM }}$ bei größeren Patientenkollektiven werde derzeit in mehreren nicht-interventionellen Studien (READOUTsmart, RETAIN-smart, SMART) untersucht, die Daten zur Injektionstreue aus der Speicherfunktion des elektronischen Injektors beziehen.

Die auf dem diesjährigen ENS-Meeting vorgestellten 15-Jahresdaten zur Therapie mit Interferon-beta 1a s.c. (Rebif ${ }^{\oplus}$ ) rief Professor Peter Rieckmann, Chefarzt, Sozialstiftung - Klinikum Bamberg, in Erinnerung. Die PRISMS-15-Studie habe den langfristigen klinischen Nutzen der höheren Dosierung (3 x 44 mg/Woche) und hochfrequenten Applikation von Interferon beta-1a s.c. gezeigt [Kappos L et

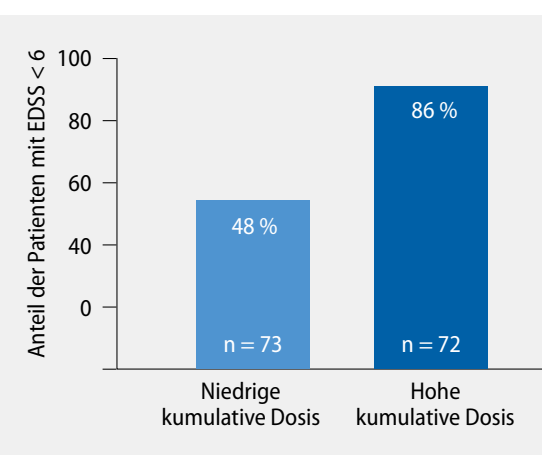

1 Nach 15 Jahren bleiben in der Hochdosierungsgruppe neun von zehn Patienten gehfähig.

al. 22nd ENS-Meeting, 2012, Prag, P470]. Sie führe zu einer verbesserten Prognose hinsichtlich Schüben, EDSS-Progression ( $\vee$ Abb. 1) und dem Übergang in eine sekundär progrediente MS.

Dr. Gunter Freese, Springer Medizin

Pressegespräch „3 Jahre RebiSmart“, München, 18.9.2012; Veranstalter: Merck Serono

\section{Bipolare Störungen}

\section{Potenzial zum Stimmungsstabilisierer}

Bipolare Störungen gehören mit ihrer hohen Krankheitsbelastung, häufiger Komorbidität und oft kompliziertem Verlauf zu den facettenreichsten Erkrankungen in der Psychiatrie, betonte Professor Volker Arolt, Münster. Zu den größten Herausforderungen gehören laut Arolt Phasenumschläge, Rapid Cycling, unvollständige Remission mit belastenden Residualsymptomen, Chronifizierung und Suizidalität.

Ein ideales „Anti-Bipolaricum“, so Arolt, wäre ein Stimmungsstabilisierer, der die Ausschläge der Gemütslage in einem nicht pathologischen Schwankungsbereich halte. Neben Lithium und Valproat werden inzwischen auch Antipsychotika der zweiten Generation in der Therapie bipolarer Störungen eingesetzt. Von diesen habe bislang nur für Quetiapin eine antimanische, antidepressive und phasenprophylaktische Wirkung gezeigt werden können. Dass auch das neue Antipsychotikum der zweiten Generation Asenapin $\left(\right.$ Sycrest $\left.^{\oplus}\right)$, bislang nur für manische Phasen der bipolaren Störung zugelassen, das Potenzial für einen echten Stimmungsstabilisierer besitzt, machte Arolt anhand von Studiendaten deutlich. So seien in der APOLLO-12-Studie [Szegedi A et al. J Clin Psychopharmacol 2012; 32: 46-55] durch die Gabe von Asenapin $(5 \mathrm{mg}$ oder $10 \mathrm{mg} 2 \mathrm{x} / \mathrm{d}$ ) zu Lithium oder Valproat sowohl der Anteil der Responder als auch der Anteil der Remitter im Vergleich zu Placebo plus Lithium oder Valproat signifikant gestiegen (YMRS Woche 12: Responder $47,7 \%$ vs. $34,4 \%$, Remitter $43,2 \%$ vs. $30,1 \%$, je $\mathrm{p}<0,05)$.

Die Wirksamkeit auf depressive Symptome sei unter anderem in einer Post-hocAnalyse von Azorin et al. [Affect Disord 2013; 145: 62-9] gezeigt worden. Darin war der MADRS-Gesamtwert nach drei Wochen signifikant stärker gesunken als unter Placebo und auch etwa 1,7 Punkte stärker als unter der aktiven Vergleichssubstanz Olanzapin. Nach zwölf Wochen war der nummerische Unterschied $\mathrm{zu}$ Olanzapin noch stärker ausgeprägt (4 Punkte), allerdings nach wie vor nicht signifikant. Eine signifikante Überlegenheit von Asenapin gegenüber Placebo zeigte sich in der Verbesserung der Items „Unfähigkeit zu fühlen“, „reduzierter Appetit“ und „innere Anspannung“. Bei letzterem Item war Asenapin auch Olanzapin signifikant überlegen.

Dr. Gunter Freese, Springer Medizin

Satellitensymposium „Unipolare Depression und bipolare Manie erfolgreich behandeln - ein Update", DGPPN-Kongress 2012, Berlin, 23.11.2012; Veranstalter: Lundbeck 